\title{
Multidimensional Cross Parity Check Codes as a Promising Solution to CubeSat Low Data Rate Downlinks
}

\author{
Ivo Vertat \\ Faculty of Electrical Engineering \\ University of West Bohemia \\ Pilsen, Czech Republic \\ ivertat@kae.zcu.cz
}

\author{
Ludek Dudacek \\ Faculty of Electrical Engineering \\ University of West Bohemia \\ Pilsen, Czech Republic \\ dudacek1@kae.zcu.cz
}

\begin{abstract}
Simple cross parity check codes have been well known for decades in the areas of magnetic recording and radiation-hardened memory in space applications. However, the computational power requirements of higher dimensional cross codes mean that priority has been given to different forward error correction methods, with the result that cross parity check codes have not become sufficiently generalized to include a higher number of dimensions. In the last two decades, the focus has mainly been on Reed-Solomon codes, Hamming codes, BCH codes, convolutional codes and turbo codes. In this paper, the issues behind generalized multidimensional parity check codes are explored again in relation to current main-stream interest by covering parity check codes in the form of low-density parity checks. I will put forward a new solution to the multidimensional parity check code decoding process for low data rate downlinks in CubeSat satellites. I propose that the high computational power of software-defined radio can be utilized for iterative decoding of CubeSat satellite transmissions in ground control segments, ensuring that onboard multidimensional parity encoders are as simple to use as possible.
\end{abstract}

Keywords - forward error correction code; cross parity check code, multidimensional parity check code; product code; iterative decoding; CubeSat

\section{INTRODUCTION}

CubeSat satellites are widely used as a cheap carrier as a means of demonstrating and verifying new in-orbit technology. Radio transmission from the CubeSat is commonly realized via amateur radio frequency bands and low-output radiated power (with typical radiated power of less than $500 \mathrm{~mW}$ ) due to the reduced power generated by satellite solar cells. This factor as well as usage of non-directional antennae on satellites, satellite attitudes without active control, narrow band channels and strong interferences all only lead to low data rates in downlink directions (from satellites to ground segments). Low data throughput may not be critical, because many CubeSat experiments do not require much data for transmission. However, for amateur radio frequency bands that encounter frequent interference, robust forward error correction methods are needed to keep the bit error rate as low as is necessary for proper scientific and basic telemetric data interpretation.
Firstly, producing a reliable forward error correction method is complicated due to the hardware and software limitations of CubeSat satellite systems (high density memory and powerful processors with very large scale integration are unreliable due to cosmic radiation effects). The onboard forward error correction encoder must be operable using simple lowpowered hardware even for long block code lengths. On the other hand, there are no limitations with the ground segment forward error correction decoder, which can be run via real-time processing used by software-defined radio as well as off-line processing.

The second complication in delivering reliable error correction methods arises from interferences in amateur radio channel usage, where long burst errors can occur. Long burst errors can also be produced by fading due to the free rotation of satellite antennae during radio transmission. In these cases, the bit error rate increases by several orders, meaning that many low robust error correction methods may then make more errors on the output when the high bit error rate is present on the input of the decoder. This may occur even when an interleaving technique is used to break the long error burst into isolated error bits.

The coding gain's ability to be easily adapted is another important requirement, because CubeSat technology only offers limited reliability. Forward error correction methods must be able to change the code rate over a wide range as a means of overcoming failures in some systems, such as antenna deployment, attitude control and power supply and in order to at least maintain emergency telemetric transmission.

Currently, state-of-the-art forward error correction methods revert to simple block codes that use advanced decoding techniques, good examples of which are low-density parity check codes (LDPC) based on iterative decoding from soft-decision demodulator outputs. LDPC were introduced in 1963 by [1], but they were applied many years later. For specialist applications, product codes may be used [2]; product codes were discovered in 1954 by [3]. These codes commonly use data arranged into a twodimensional matrix and in each dimension some type of correction or detection code is used, examples of which can be found in [4] and [5].

However, sufficient results for CubeSat transmission (a required bit error rate of $10^{-5}$ ) can be 
achieved with a simpler product code, based only on the cross parity check codes that become generalized in a multidimensional space. These codes are described in [6] and in this paper they will be analyzed using iterative decoding from hard-decision demodulator outputs. Multidimensional parity check codes will fully satisfy all mentioned requirements, including simple encoder realization, robustness of high bit error rates at the decoder input as well as adaptation with a wide range of coding gains.

The rest of the paper is organized as follows: Chapter II describes generalized multidimensional cross parity check codes, graphical representations of codes in a multidimensional space and basic equations. Proposed iterative hard decision-based decoding algorithms will be outlined in chapter III. Chapter IV details the results of bit error rate and frame error rate simulations using an AWGN (additive white Gaussian noise) channel. Results and possible future work in this area is laid out in the last chapter.

\section{MULTIDIMENSIONAL CROSS PARITY CHECK CODES}

In common two-dimensional cross parity check codes, data bits are arranged into a matrix of columns and rows. Column parity bits are placed in the last row. Each parity bit in this row secures data bits in the column above. Row parity bits are placed in the last column. Each parity bit in this column secures data bits beside it. The parity bit in the bottom right corner is called a check bit on check bits and secures column and row parity bits. These codes are shown in the left part of Fig. 1.This solution and some enhancements are described in many references, e.g. [7], [8], [9] and [10]. Such codes are suitable only for low bit error rates at the input of the FEC (forward error correction) decoder due to high sensitivity to multiple errors in the same rows or columns. However, it allows a higher code rate to work even with small codeword lengths in comparison to higher dimensionality parity codes. Small codeword length can be an important requirement in some applications with limited memory capacity or limited delay.

Generalized multidimensional parity check codes can be constructed by arranging the basic three dimensional cross parity check cubes into a multidimensional hypercube. A three-dimensional cross parity cube (right part of Fig. 1) consists of twodimensional cross parity matrices layered into a third dimension. The last layer contains the parity bits across the layers of data bits. A four-dimensional cross parity check code can be constructed by composing three-dimensional cubes into one row. A fivedimensional cross parity check code is created by arranging three-dimensional cubes into rows and columns (Fig. 2) and a six-dimensional cross parity check code is created by layering five-dimensional structures (Fig. 3). An introduction to multidimensional parity check codes can be found in [11].

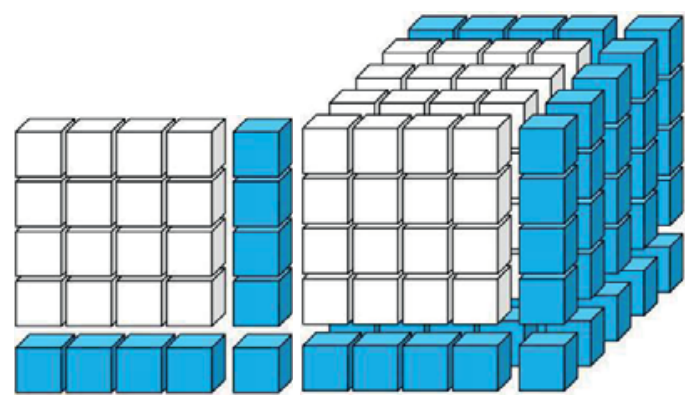

Fig. 1 A geometric representation of data bits (white box) and parity bits (blue box) for cross parity check codes in two dimensions (left) and in three dimensions (right).

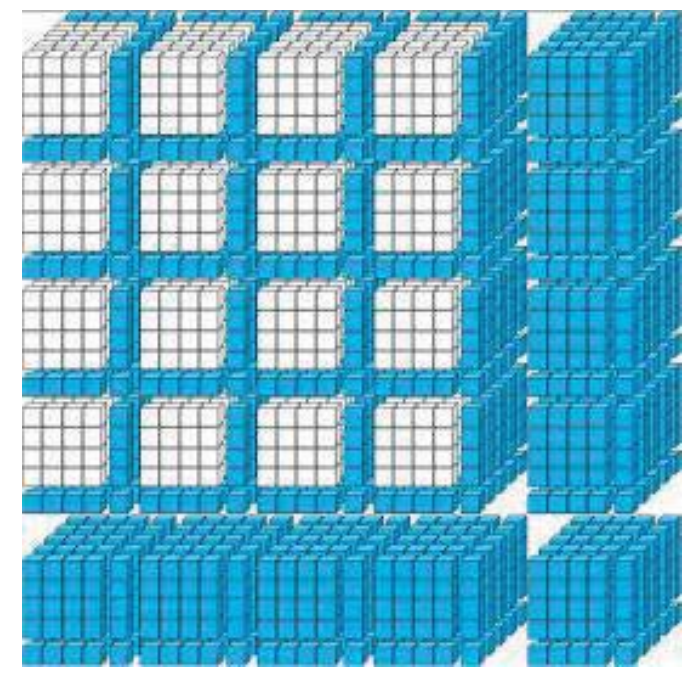

Fig. 2 A generalized representation of multidimensional cross parity check codes by concatenating three-dimensional cubes into fivedimensional structure.

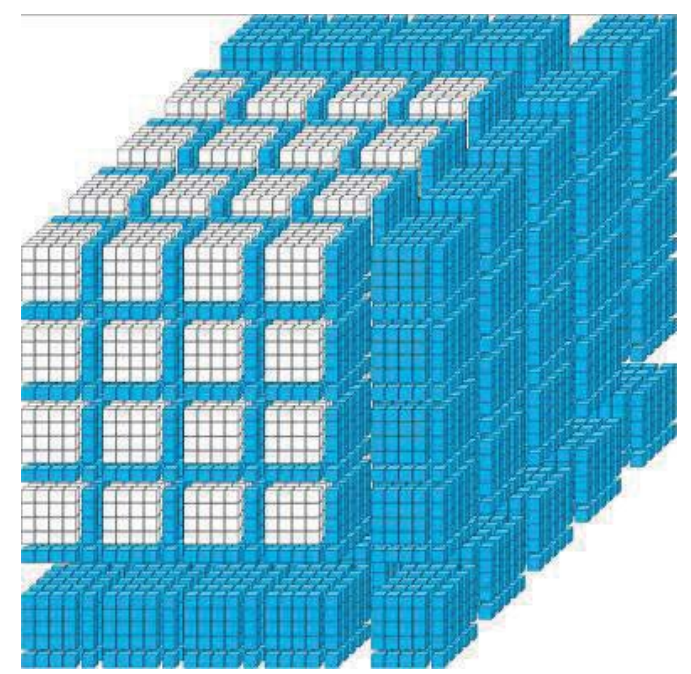

Fig. 3 A generalized representation of multidimensional cross parity check codes by concatenating three-dimensional cubes into sixdimensional structure.

In the following text, multidimensional cross parity check codes will be labeled $\operatorname{MDPC}(x \mathrm{D} / y \mathrm{~L})$, where $x$ is the number of dimensions (D) and $y$ is the length (L) of data bits in each dimension. Fig. 4 shows relations between data bits and parity bits for MDPC $(5 \mathrm{D} / 4 \mathrm{~L})$ codes. In this case, there is shown code with 5 dimensions and 4 data bits in each dimension. Each parity bit secures 4 data bits and each data bit is secured by 5 parity bits. 


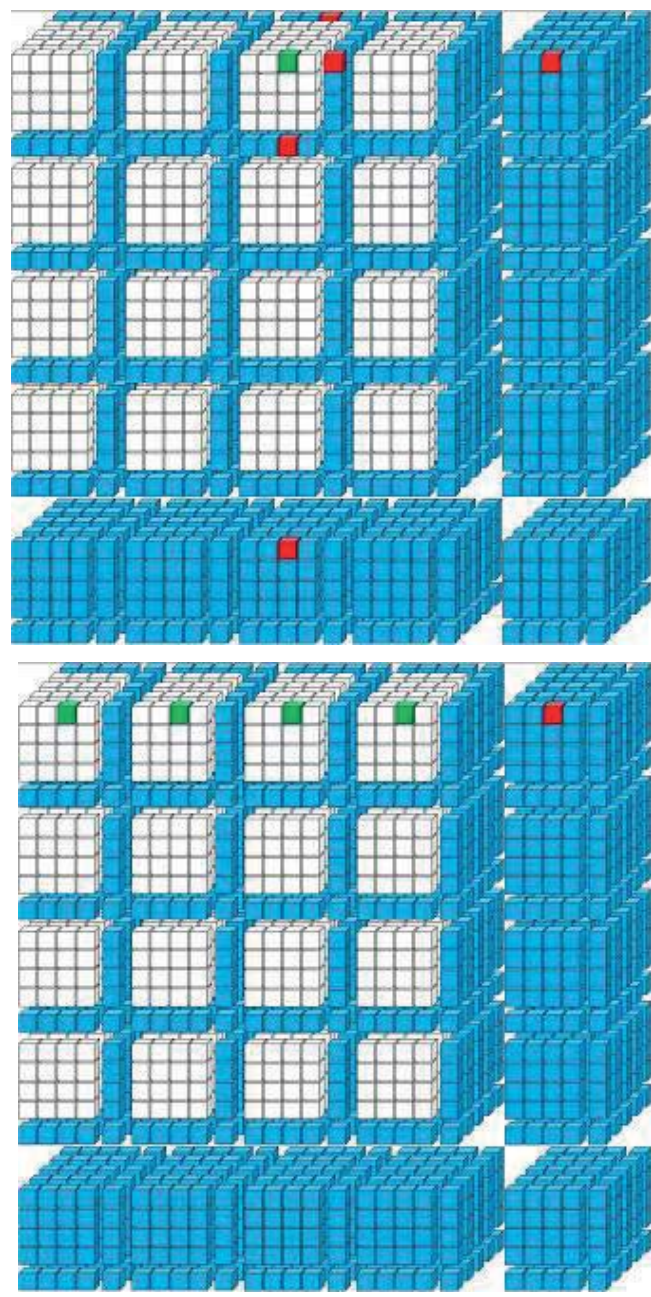

Fig. 4 The relation of data bits and parity bits in a five-dimensional space. The upper part shows the position of 5 parity bits (red) which secure 1 specific data bit (green) and the lower part shows the position of 4 data bits (green) secured by 1 specific parity bit (red).

In arbitrary $\operatorname{MDPC}(x \mathrm{D} / y \mathrm{~L})$ code, each parity bit secures $y$ data bits and $x$ parity bits secure each data bit. The number of data bits at the encoder input (message word length) can be calculated as:

$$
k=y^{x}
$$

The total number of bits in a secured block of data at the encoder output (codeword length) can be calculated as:

$$
n=(y+1)^{x}
$$

The code rate of the $\operatorname{MDPC}(x \mathrm{D} / y \mathrm{~L})$ code can be calculated as:

$$
R=\frac{k}{n}=\frac{y^{*}}{(y+1)^{*}}
$$

Equations 1, 2 and 3 clearly show the practical limitations of multidimensional cross parity check codes. As the number of dimensions increases to better secure the data bits and the length of data bits in each dimension stays constant, the code rate falls down. A higher code rate can be maintained by increasing the data bit length; however, both the encoder and decoder must work with a very long codeword, which has a negative impact on memory usage and processing delay. Table 1 shows the code rate as a function of dimensionality and data bit length in each dimension. Table 2 shows the codeword length as a function of

\begin{tabular}{|c|c|c|c|c|c|c|}
\hline & \multicolumn{5}{|c|}{$x$-number of dimensions } \\
\hline & & 3 & 4 & 5 & 6 & 7 \\
\hline \multirow{9}{*}{ 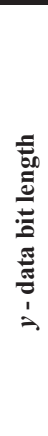 } & 1 & 0.125 & 0.063 & 0.031 & 0.016 & 0.008 \\
\hline & 2 & 0.296 & 0.198 & 0.132 & 0.088 & 0.059 \\
\hline & 3 & 0.422 & 0.316 & 0.237 & 0.178 & 0.133 \\
\hline & 4 & 0.512 & 0.410 & 0.328 & 0.262 & 0.210 \\
\hline & 5 & 0.579 & 0.482 & 0.402 & 0.335 & 0.279 \\
\hline & 6 & 0.630 & 0.540 & 0.463 & 0.397 & 0.340 \\
\hline & 7 & 0.670 & 0.586 & 0.513 & 0.449 & 0.393 \\
\hline & 8 & 0.702 & 0.624 & 0.555 & 0.493 & 0.438 \\
\hline & 9 & 0.729 & 0.656 & 0.590 & 0.531 & 0.478 \\
\hline
\end{tabular}
dimensionality and data bit length in each dimension.

Tab. 1 Code rates for several variants of $\operatorname{MDPC}(x \mathrm{D} / y \mathrm{~L})$.

Tab. 2 Codeword lengths for several variants of $\operatorname{MDPC}(x \mathrm{D} / y \mathrm{~L})$.

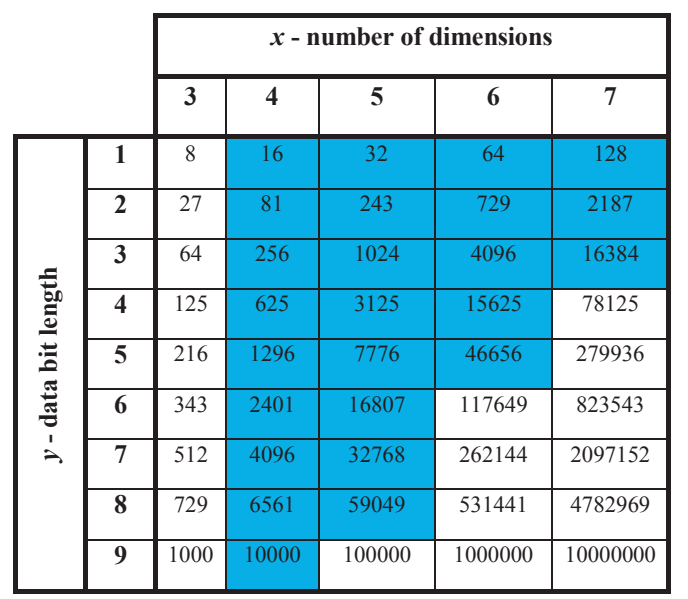

If we consider a codeword length of $2^{16}$ as adequate for manageable memory size and at least 4 dimensions for better securing of data bits, then the blue color-marked variants of MDPC codes from Table 1 and Table 2 offer a very wide range of code rates - from 0.656 (close to a common code rate of $2 / 3$ ) to 0.00015 (not presented in tables; 16 dimensions and 1 data bit length are used) - for highly secured data transmission.

\section{PROPOSED ITERATIVE DECODING ALGORITHM}

Two-dimensional cross parity check codes cannot apply any more sophisticated rules to the decoding process. If the column parity check and row parity check do not pass at the same time, an error is detected in the intersection position of the failed row and failed column and bit value is inverted. If only the row parity check or column parity check do not pass, the error is detected; however, it cannot be corrected due to the unknown position of the failed bits. This can happen if one of two parity check dimensions is improperly evaluated due to multiple errors. 
In multidimensional (three-dimensional and more) cross parity checks, the decoding algorithm can redundantly secure data bits by placing parity bits in more than two dimensions. For example, in $\operatorname{MDPC}(5 \mathrm{D} / 4 \mathrm{~L})$ the parity check process for a particular bit can fail in 1 dimension, 2 dimensions, 3 dimensions, 4 dimensions or in all 5 dimensions. This means that in the last two cases there is a high probability of error at given secured bit position, after which the bit value is flipped. If the parity check fails only in 2 or 3 dimensions, the error in the given position is not as conclusive and the decoding algorithm must carefully consider flipping the bit value. Iterative decoding can be a strong method for error correction in such a case.

This proposed iterative decoding method corrects the bits with the highest probability of error in each decoding iteration. Each decoding iteration starts by checking all parity bits and their related data bits. For each bit (data and parity), a failed dimension marker (FDM) is counted. If an arbitrary bit discovers a parity check error in the $k$ dimension, its FDM is set to $k$. For $\operatorname{MDPC}(5 \mathrm{D} / 4 \mathrm{~L}), k$ can exist in a range of 0 to 5 . Decoding stops if the highest FDM value is less than 2 (no correctable errors); otherwise, all bits with the highest FDM are inverted. In the next step, the iteration number limit (INL) is checked. If the predefined maximum iteration (in a simulation set to 200) is not reached, all steps are repeated in the next iteration. If the INL is reached, the decoding stops.

Error frame detection is based on the highest FDM value $\left(\mathrm{FDM}_{\max }\right)$ once decoding ends. If the $\mathrm{FDM}_{\max }$ is equal to 0 after decoding, the frame is considered error-free. If the $\mathrm{FDM}_{\max }$ is equal to 1 or higher after decoding, the frame is considered an uncorrectable error. All decoding steps are shown in the flow-chart diagram in Fig. 5.

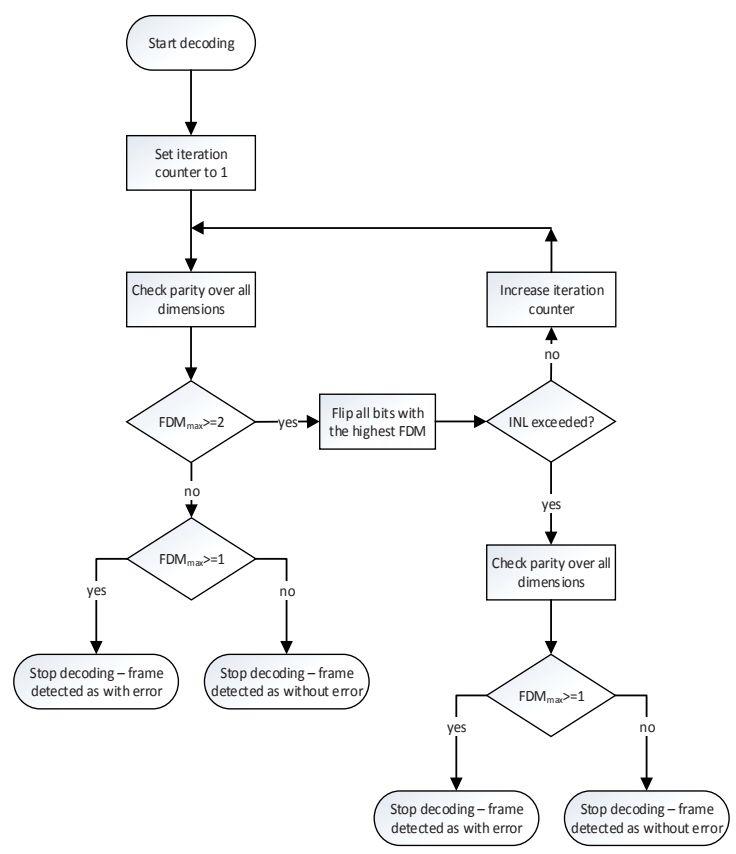

Fig. 5 Flow-chart diagram of the first iterative decoding algorithm.

\section{BIT ERROR RATE PERFORMANCE RESULTS}

The two main decoding method output parameters - the bit error rate (BER) and the frame error rate (FER) - were analyzed using an AWGN channel model. The BER is the ratio between the decoded bits containing error and the total amount of decoded bits; the FER is the ratio between the number of decoded frames containing error and the total number of decoded frames.

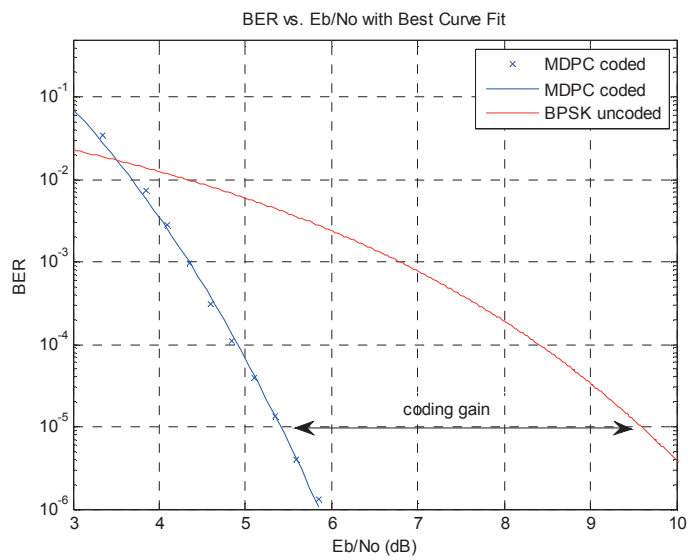

Fig. 6 Bit error rate performance of the proposed hard decisionbased iterative decoding of the MDPC $(5 \mathrm{D} / 4 \mathrm{~L})$ code with a code rate of $\mathrm{R}=1024 / 3125$.

For CubeSat satellites, a BER equal to $10^{-5}$ is commonly used in power link budgets. In such a case, the proposed iterative method applied to the MDPC $(5 \mathrm{D} / 4 \mathrm{~L})$ code with a code rate equal to $1024 / 3125$ offers a coding gain equal to $4.1 \mathrm{~dB}$ and requires that the $\mathrm{E}_{\mathrm{b}} / \mathrm{N}_{0}$ (energy per bit to noise power spectral density ratio) equal $5.4 \mathrm{~dB}$ for coherent BPSK (binary-phase shift keying) demodulation. Complete BER results are shown in Fig. 6.

\section{CONCLUSION}

Cross parity check codes that are extended to include a higher number of dimensions can offer simple encoding suitable for power limited hardware used in CubeSat satellites. Simulation results show a positive coding gain even for simple hard decision decoding (approximately $4.1 \mathrm{~dB}$ for a $\mathrm{BER}=10^{-5}$ ) in comparison to uncoded coherent BPSK using an AWGN channel for the MDPC $(5 \mathrm{D} / 4 \mathrm{~L})$ code, which has a code rate close to $1 / 3$ and a manageable short codeword length (3125 bits).

A simple multidimensional parity encoder can be formed by addressing memory and modular arithmetic onboard the CubeSat satellites. Once manageable requirements on memory size are respected, decoding can be run on a common personal computer with demodulated output from widely used softwaredefined radios. My current work is an investigation of soft decision iterative decoding of multidimensional parity codes. Preliminary results show significant improvements in comparison to hard decision-based decoding. 
The results of simple hard decision-based iterative decoding foresee the great potential of MDPC when using soft decision-based iterative decoding. There is also possibility to use MDPC codes together with $\mathrm{CRC}$ (cyclic redundancy check) as rate-less codes and additional parity bits in higher dimensions transmits only on request if decoding failed. MDPC should attract the same attention as low-density parity codes in light of new signal processing methods and the more powerful hardware; with properties which had not been discovered in the era of their widespread use in the form of only two dimensional cross parity codes for data storage.

\section{ACKNOWLEDGMENT}

This project was funded by the SGS-2018-001.

\section{REFERENCES}

[1] R. G. GALLAGER, "Low-Density Parity-Check Codes." Cambridge, MA:MIT Press, 1963.

[2] C. CARLO, "Concatenated Turbo/LDPC codes for deep space communications: performance and implementation." In SPACOMM 2013, The Fifth International Conference on Advances in Satellite and Space Communications, pp. 1-6. 2013.

[3] P. ELIAS, "Error free coding," IRE Trans. Inform. Theory, vol. IT-4, pp. 29-37, Sept. 1954.

[4] M. POOLAKKAPARAMBIL, J. MATHEW, A. M. JABIR, S. P. MOHANTY, "Low complexity cross parity codes for multiple and random bit error correction," Quality Electronic Design (ISQED), 2012 13th International Symposium on, vol., no., pp.57,62, 19-21 March 2012. doi: 10.1109/ISQED.2012.6187474

[5] Y. CASSUTO, M. A. SHOKROLLAHI, "Array-code ensembles -or- two-dimensional LDPC codes," Information Theory Proceedings (ISIT), 2011 IEEE International Symposium on , vol., no., pp.518,522, July 31 2011-Aug. 5 2011. doi: 10.1109/ISIT.2011.6034181

[6] D. M. RANKIN, T. A. GULLIVER, "Single parity check product codes," Communications, IEEE Transactions on, vol.49, no.8, pp.1354,1362, Aug 2001. doi: 10.1109/26.939851

[7] Q. L. RAO, C. HE, "A new 2-D parity checking architecture for radiation-hardened by design SRAM," Microelectronics \& Electronics, 2009. PrimeAsia 2009. Asia Pacific Conference on Postgraduate Research in , vol., no., pp.360,363, 19-21 Jan. 2009. doi: 10.1109/PRIMEASIA.2009.5397372

[8] D. AFLAKIAN, et al., "Error Detection and Correction over Two-Dimensional and Two-Diagonal Model and FiveDimensional Model." International Journal of Advanced Computer Science \& Applications 2.7 (2011).

[9] A. FELTSTROM, J. D. TRUHACHEV, M. LENTMAIER, K. S. ZIGANGIROV, "Braided block codes." Information Theory, IEEE Transactions on 55, no. 6 (2009): 2640-2658.

[10]A. VADINALA, G. K. KUMAR, "Multi Dimensional Parity Based Hamming Codes For Correcting The SRAM Memory Faults Under High Emi Conditions." In IACEECE International Conference, pp.46-49. 2013

[11]J. M. SHEA, T. F. WONG, "Multidimensional Codes." Encyclopedia of Telecommunications. Wiley 2003. 